\title{
Co-spin with Symmetry Axis Stabilization, and De-spin for Asteroid Capture
}

\author{
Haijun Shen ${ }^{\dagger}$ and Carlos M. Roithmayr ${ }^{\ddagger}$
}

\begin{abstract}
Consideration is given to attitude control associated with capturing a free-flying asteroid using an axisymmetric spacecraft. Asymptotically stable controllers are designed to align the spacecraft axis of symmetry with a line of descent that is fixed in the asteroid, and to eliminate all relative angular velocity before capture takes place. An analytical expression is presented for the torque required to maintain alignment of the axes of symmetry of the spacecraft and an axisymmetric asteroid. After the asteroid is securely captured, the angular velocity of the rigid composite body relative to an inertial frame is arrested; we present a controller that is asymptotically stable and stays within specified thrust limits.
\end{abstract}

\section{INTRODUCTION}

Capturing and returning an asteroid to the Earth-Moon system has garnered a great deal of attention recently (Ref. [1]). References [2] and [3] discuss various phases of the operations when the spacecraft is in the vicinity of the asteroid, including initial approach, hover, spacecraft spinup, descent, ascent (if a small boulder is picked up from a large asteroid), and de-spin. Dynamics and control in each of these activities is analyzed in order to determine the velocity increments and control force and torque that must be provided by a Reaction Control System (RCS), and the mass of the propellant that will be consumed.

This paper addresses in depth the attitude control required for spin-up and de-spin. First, the spacecraft is reoriented and spun up so that it points to the asteroid properly, and all angular velocity relative to the asteroid is eliminated to ensure capture of the asteroid. Second, the body consisting of the spacecraft and the captured asteroid is de-spun to eliminate angular velocity relative to an inertial reference frame, to prepare for the return to the Earth-Moon system.

We make the following assumptions. The asteroid is a rigid body with mass distributed in the most general possible way; that is, it can possess three distinct principal moments of inertia, and is therefore referred to as unsymmetric. Before capture the asteroid undergoes torque-free motion and in general can tumble, and its angular velocity and mass properties are known. The spacecraft is an axisymmetric rigid body. The capture mechanism is also axisymmetric; the axes of symmetry of the spacecraft and capture mechanism are collinear. Due to symmetry, effective use of the capture mechanism does not require any particular relative orientation, about the axis of symmetry, between the spacecraft and the asteroid. The spacecraft approaches the asteroid along a straight line (line of descent) that is fixed in the asteroid, as illustrated in Fig. 1.

\footnotetext{
${ }^{\dagger}$ Analytical Mechanics Associates, Inc., 21 Enterprise Parkway, Suite 300, Hampton, VA 23666, USA +1 757865 0000, shen@ama-inc.com

‡NASA Langley Research Center, Vehicle Analysis Branch, MS 451, 1 North Dryden Street, Hampton, VA, 23681, USA +1 757864 6778, carlos.m.roithmayr@nasa.gov
}

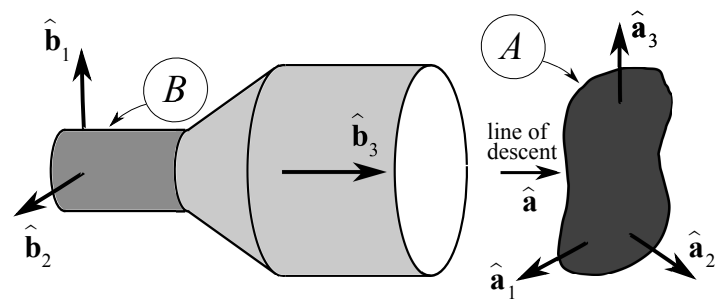

Fig. 1. Illustration of spacecraft approaching asteroid.

Here, we take the approach of removing completely the relative angular velocity between the spacecraft and the asteroid, based on the assumption that this offers the best chance to avoid damage or destruction of the mechanism when capture takes place. Thus, the objectives of spin-up control are to make the axis of symmetry of the spacecraft parallel to the line of descent, and to eliminate rotational motion of the spacecraft relative to the asteroid about the line of descent.

We assume that, after capture, the asteroid is rigidly connected to the spacecraft so that together they form one rigid body, which is considered unsymmetric to maintain generality. The body must undergo de-spin to eliminate angular velocity relative to the inertial frame. This is necessary, for example, to orient solar arrays properly or to aim thrust in the direction needed to return to the Earth-Moon system. A controller is designed to be asymptotically stable, and stay within specified thruster limits.

The paper is organized as follows. The controllers that align the axis of symmetry of the spacecraft with the line of descent, and eliminate relative angular velocity between the two bodies, are presented first. Next, an analytical expression is presented for the control torque required to maintain alignment of the axes of symmetry of the spacecraft and the special case of an axisymmetric asteroid. Finally, the despin controller is presented, followed by conclusions and references.

\section{ATtitude SynCHRONIZATION}

In this section we present controller designs that facilitate the synchronization of the spacecraft axis of symmetry and the line of descent. To this end, consider a unit vector $\hat{a}$ fixed in the asteroid, $A$, parallel to the line of descent, and having a direction consistent with the descent. Let $\hat{\mathbf{b}}_{1}, \hat{\mathbf{b}}_{2}$, and $\hat{\mathbf{b}}_{3}$ be a set of right-handed, mutually orthogonal unit vectors fixed in the spacecraft, $B$, with $\hat{\mathbf{b}}_{3}$ parallel to the axis of symmetry, and marking the direction in which the spacecraft is to advance toward the asteroid. It is desired to design a controller to align $\hat{\mathbf{b}}_{3}$ with $\hat{\mathbf{a}}$, and to eliminate 
all relative rotational motion before capture takes place, without requiring any particular relative orientation between the spacecraft and the asteroid about $\hat{\mathbf{b}}_{3}$.

\section{A. Kinematics and Dynamics}

The direction of $\hat{\mathbf{a}}$ with respect to the spacecraft reference frame is described with two parameters, $w_{1}$ and $w_{2}$, obtained through stereographic projection as discussed in Ref. [4] and illustrated in Fig. 2. If the unit vector $\hat{\mathbf{a}}$ is written as

$$
\hat{\mathbf{a}}=a \hat{\mathbf{b}}_{1}+b \hat{\mathbf{b}}_{2}+c \hat{\mathbf{b}}_{3}
$$

then $w_{1}$ and $w_{2}$ are defined as

$$
w_{1}=\frac{b}{1+c} \quad w_{2}=\frac{-a}{1+c}
$$

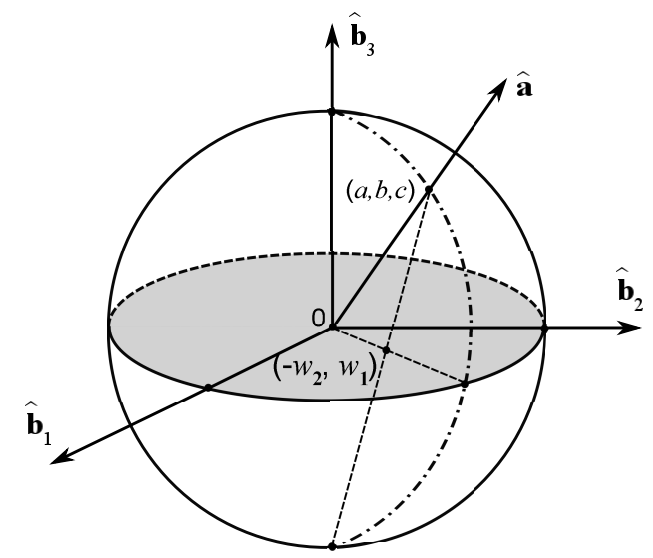

Fig. 2. Illustration of stereographic projection and parameters $w_{1}$ and $w_{2}$.

As illustrated in Fig. 2, when $w_{1}=w_{2}=0, \hat{\mathbf{b}}_{3}$ has the same direction as $\hat{\mathbf{a}}$, implying that the axis of symmetry is aligned with the line of descent. Thus, the objective of the controller design is to drive both $w_{1}$ and $w_{2}$ to zero, and to eliminate all relative angular velocity between $B$ and $A$.

The problem of pointing the axis of symmetry, $\hat{\mathbf{b}}_{3}$, of an axisymmetric spacecraft in the inertial frame is studied in Ref. [5], where kinematic equations are given that relate the angular velocity of the spacecraft to the rates of $w_{1}$ and $w_{2}$, as well as several controllers that either exponentially or asymptotically stabilize $\hat{\mathbf{b}}_{3}$. The problem in this paper is different in that we are dealing with the orientation of $\hat{b}_{3}$ in $A$, which is a rotating frame.

Let ${ }^{A} \boldsymbol{\omega}^{B}$ denote the angular velocity of the spacecraft relative to the asteroid. Since â is fixed in frame $A$,

$$
\frac{{ }^{A} \mathrm{~d} \hat{\mathbf{a}}}{\mathrm{d} t}={ }^{B} \frac{\mathrm{d} \hat{\mathbf{a}}}{\mathrm{d} t}+{ }^{A} \boldsymbol{\omega}^{B} \times \hat{\mathbf{a}}=0
$$

where ${ }^{X} \frac{\mathrm{d}(\cdot)}{\mathrm{d} t}$ denotes the derivative of a vector with respect to time taken in a reference frame $X$. In addition, Eq. (1) leads to

$$
{ }^{B} \frac{\mathrm{d} \hat{\mathbf{a}}}{\mathrm{d} t}=\dot{a} \hat{\mathbf{b}}_{1}+\dot{b} \hat{\mathbf{b}}_{2}+\dot{c} \hat{\mathbf{b}}_{3}
$$

It can be shown from Eqs. (2), (3), and (4) that $w_{1}$ and $w_{2}$ are governed by the following kinematic equations.

$$
\begin{aligned}
& \dot{w}_{1}=w_{2} \delta_{3}+\delta_{2} w_{1} w_{2}+\frac{\delta_{1}}{2}\left(1+w_{1}^{2}-w_{2}^{2}\right) \\
& \dot{w}_{2}=-w_{1} \delta_{3}+\delta_{1} w_{1} w_{2}+\frac{\delta_{2}}{2}\left(1+w_{2}^{2}-w_{1}^{2}\right)
\end{aligned}
$$

where

$$
\delta_{i}={ }^{A} \boldsymbol{\omega}^{B} \cdot \hat{\mathbf{b}}_{i} \quad(i=1,2,3)
$$

are the measure numbers (Ref. [6], p. 3) of ${ }^{A} \boldsymbol{\omega}^{B}$ for $\hat{\mathbf{b}}_{1}$, $\hat{\mathbf{b}}_{2}$, and $\hat{\mathbf{b}}_{3}$.

The dynamics of the rotational motion of the spacecraft are described by Euler's equations. Let $I_{i}(i=1,2,3)$ denote the principle moments of inertia of the spacecraft. For an axisymmetric body, $I_{1}=I_{2}$. Let

$$
\omega_{i}={ }^{N} \boldsymbol{\omega}^{B} \cdot \hat{\mathbf{b}}_{i} \quad(i=1,2,3)
$$

denote the $\hat{\mathbf{b}}_{1}-\hat{\mathbf{b}}_{2}-\hat{\mathbf{b}}_{3}$ measure numbers of the angular velocity of spacecraft $B$ relative to an inertial frame $N$. Then, Euler's equations are written as

$$
\begin{aligned}
& \dot{\omega}_{1}=a_{1} \omega_{2} \omega_{3}+u_{1} \\
& \dot{\omega}_{2}=-a_{1} \omega_{3} \omega_{1}+u_{2} \\
& \dot{\omega}_{3}=u_{3}
\end{aligned}
$$

where $a_{1}=\left(I_{2}-I_{3}\right) / I_{1}$ and $u_{i}=M_{i} / I_{i}(i=1,2,3)$. Here, $M_{1}, M_{2}$, and $M_{3}$ are the $\hat{\mathbf{b}}_{1}-\hat{\mathbf{b}}_{2}-\hat{\mathbf{b}}_{3}$ measure numbers of the torque acting on the spacecraft.

The dynamic equations that govern the relative angular velocity measure numbers $\delta_{i}(i=1,2,3)$ can then be derived. By definition in Eq. (6),

$$
\delta_{i}=\left({ }^{N} \boldsymbol{\omega}^{B}-{ }^{N} \boldsymbol{\omega}^{A}\right) \cdot \hat{\mathbf{b}}_{i} \quad(i=1,2,3)
$$

Let

$$
\varpi_{i}={ }^{N} \boldsymbol{\omega}^{A} \cdot \hat{\mathbf{b}}_{i} \quad(i=1,2,3)
$$

denote the $\hat{\mathbf{b}}_{1}-\hat{\mathbf{b}}_{2}-\hat{\mathbf{b}}_{3}$ measure numbers of the angular velocity of the asteroid relative to the inertial frame. Then,

$$
\delta_{i}=\omega_{i}-\varpi_{i} \quad(i=1,2,3)
$$

Eqs. (8) and (10) together yield

$$
\begin{aligned}
& \dot{\delta}_{1}=a_{1} \delta_{2} \delta_{3}+v_{1} \\
& \dot{\delta}_{2}=-a_{1} \delta_{3} \delta_{1}+v_{2} \\
& \dot{\delta}_{3}=v_{3}
\end{aligned}
$$

where $v_{i}(i=1,2,3)$ are new control variables that are related to the original control variables $u_{i}(i=1,2,3)$ by

$$
\begin{aligned}
& v_{1}=u_{1}+a_{1}\left(\varpi_{2} \varpi_{3}+\delta_{2} \varpi_{3}+\varpi_{2} \delta_{3}\right)-\dot{\varpi}_{1} \\
& v_{2}=u_{2}-a_{1}\left(\varpi_{1} \varpi_{3}+\delta_{1} \varpi_{3}+\varpi_{1} \delta_{3}\right)-\dot{\varpi}_{2} \\
& v_{3}=u_{3}-\dot{\varpi}_{3}
\end{aligned}
$$

where $\dot{\varpi}_{i}(i=1,2,3)$ are given by

$$
\begin{aligned}
\dot{\varpi}_{i} & =\frac{\mathrm{d}}{\mathrm{d} t}\left({ }^{N} \boldsymbol{\omega}^{A} \cdot \hat{\mathbf{b}}_{i}\right) \\
& ={ }^{N} \boldsymbol{\alpha}^{A} \cdot \hat{\mathbf{b}}_{i}+{ }^{N} \boldsymbol{\omega}^{A} \cdot\left({ }^{N} \boldsymbol{\omega}^{B} \times \hat{\mathbf{b}}_{i}\right) \\
& =\left({ }^{N} \boldsymbol{\alpha}^{A}+{ }^{N} \boldsymbol{\omega}^{A} \times{ }^{N} \boldsymbol{\omega}^{B}\right) \cdot \hat{\mathbf{b}}_{i} \quad(i=1,2,3)
\end{aligned}
$$


where ${ }^{N} \boldsymbol{\alpha}^{A}$ is the angular acceleration of the asteroid relative to the inertial frame.

The asteroid is assumed to have known ratios of moments of inertia (Ref. [7]), and undergo a torque-free motion. Thus, with an attitude and angular velocity measurement at any time, the attitude, angular velocity, and angular acceleration of the asteroid can be determined at any other time from dynamical and kinematical equations of motion (Ref. [8], Secs. 3.1 and 3.4).

\section{B. Controller Design}

A close examination of Eqs. (5) and (11) reveals that the dynamics of $\delta_{3}$ is self-contained in that it is not affected by other states in the system. Thus, the problem can be separated into two subsystems, and controllers can be designed to stabilize the subsystems individually.

\section{Subsystem 1:}

$$
\begin{aligned}
\dot{\delta} & =-i a_{1} \delta_{3} \delta+v \\
\dot{w} & =-i \delta_{3} w+\frac{\delta}{2}+\frac{\bar{\delta}}{2} w^{2}
\end{aligned}
$$

\section{Subsystem 2:}

$$
\dot{\delta}_{3}=v_{3}
$$

where Subsystem 1 consists of Eqs. (5), (11a), and (11b), while Subsystem 2 consists of Eq. (11c). Here the notation of using complex numbers is adopted from Ref. [5]. Namely, $i=\sqrt{-1}, \delta=\delta_{1}+i \delta_{2}, w=w_{1}+i w_{2}, v=v_{1}+i v_{2},(\cdot)$ denotes complex conjugate, and $|\cdot|$ denotes the magnitude of a complex number.

In Ref. [5], four controllers with exponential or asymptotic behavior are developed to align $\hat{\mathbf{b}}_{3}$ with $\hat{\mathbf{n}}_{3}$, a unit vector fixed in an inertial frame. No control torque is applied about $\hat{\mathbf{b}}_{3}$; therefore, $\omega_{3}$ is constant during the time control is applied in the other two directions. Subsystem 1 resembles the system studied in Ref. [5]; in general, however, $\delta_{3}$ (the counterpart to constant $\omega_{3}$ ) is not constant. The four controllers developed in Ref. [5] are presented in Eqs. (16)-(19) with $\omega_{3}$ replaced by $\delta_{3}$. It can be shown in a straightforward way that proofs of stability given in Ref. [5] hold for $\dot{\delta}_{3} \neq 0$, and therefore remain applicable to Eqs. (16)-(19). For completeness, the four controllers for Subsystem 1 are presented as follows.

\section{Controller 1:}

$$
\begin{gathered}
v=i a_{1} \delta_{3} \delta+k_{1}\left(i \delta_{3} \delta-\frac{\delta}{2}-\frac{\bar{\delta}}{2} w^{2}\right) \\
-k_{2}\left(\delta+k_{1} w\right)
\end{gathered}
$$

with $k_{1}>0$ and $k_{2}>0$, globally asymptotically stabilizes Subsystem 1.

\section{Controller 2:}

$$
\begin{aligned}
v=i a_{1} \delta_{3} \delta & -k_{1}\left(-i \delta_{3} w+\frac{\delta}{2}+\frac{\bar{\delta}}{2} w^{2}\right) \\
& -k_{2}\left(\delta+k_{1} w\right)-w\left(1+|w|^{2}\right)
\end{aligned}
$$

with $k_{1}>0$ and $k_{2}>0$, globally exponentially stabilizes Subsystem 1 with rate of decay $\beta / 2$, where $\beta=$ $\min \left\{k_{1}, 2 k_{2}\right\}$.
Controller 3:

$$
v=-k_{1} \delta-k_{2} w
$$

with $k_{1}>0$ and $k_{2}>0$, globally asymptotically stabilizes Subsystem 1.

\section{Controller 4:}

$$
v=-k_{1} \delta-k_{2}\left(1+|w|^{2}\right) w
$$

with $k_{1}>0$ and $k_{2}>0$, globally asymptotically stabilizes Subsystem 1 .

The original control variables $u_{1}$ and $u_{2}$ can be obtained through Eqs. (12a) and (12b) as

$$
\begin{aligned}
& u_{1}=v_{1}-a_{1}\left(\varpi_{2} \varpi_{3}+\delta_{2} \varpi_{3}+\varpi_{2} \delta_{3}\right)+\dot{\varpi}_{1} \\
& u_{2}=v_{2}+a_{1}\left(\varpi_{1} \varpi_{3}+\delta_{1} \varpi_{3}+\varpi_{1} \delta_{3}\right)+\dot{\varpi}_{2}
\end{aligned}
$$

Exponential or asymptotic stabilization of Subsystem 1 ensures that $\hat{\mathbf{b}}_{3}$ becomes aligned with $\hat{\mathbf{a}}$ as time progresses. These controllers can be readily used in applications such as giving $\hat{\mathbf{b}}_{3}$ the same direction as a specified vector fixed in another rotating satellite, while allowing relative angular speed $\delta_{3}$ to vary freely. However, for retrieving an asteroid, it is desirable to drive $\delta_{3}$ to zero to avoid damaging the capture mechanism. Because of the simple form of Eq. (15) associated with Subsystem 2, it is clear that

$$
v_{3}=-k_{3} \delta_{3}
$$

with $k_{3}>0$, globally exponentially stabilizes Subsystem 2 with rate of decay $k_{3}$. From Eq. (12c), the original control variable $u_{3}$ can be obtained as

$$
u_{3}=-k_{3} \delta_{3}+\dot{\varpi}_{3}
$$

Remark: The controllers presented in this section are effective regardless of the initial angle between $\hat{\mathbf{b}}_{3}$ and $\hat{\mathbf{a}}$. However, in practice, in the cases where reorientation of $\hat{\mathbf{b}}_{3}$ can be performed without expending much propellant, it can be preferable to reorient $\hat{\mathbf{b}}_{3}$ first, spin up the spacecraft about $\hat{\mathbf{b}}_{3}$, and then apply the feedback controllers. This is because the controllers are not designed to minimize propellant usage. For example, when the spacecraft is initially at rest, a slow reorientation of $\hat{\mathbf{b}}_{3}$ can be achieved through brief thruster firings, with minimal propellant consumption, at the beginning and end of the maneuver. Additionally, the spacecraft can be spun up about $\hat{\mathbf{b}}_{3}$ once the reorientation is completed. This way, the simple attitude maneuvers are separated from more complex ones that require feedback control.

\section{CO-SPINNING AXISYMMETRIC SPACECRAFT AND AXISYMMETRIC ASTEROID}

As the axis of symmetry of the spacecraft asymptotically approaches the line of descent, torque must be applied continuously to keep the two lines collinear. An analytical expression for the torque is available for the special case of an axisymmetric asteroid and a line of descent parallel to the asteroid's axis of symmetry. During torque-free motion, the central angular momentum $\mathbf{H}$ of the asteroid $A$ in a Newtonian reference frame $N$ has constant magnitude and constant direction in $N$. The angle $\phi$ between $\mathbf{H}$ and the asteroid's axis of symmetry is constant. A reference frame $C$ 


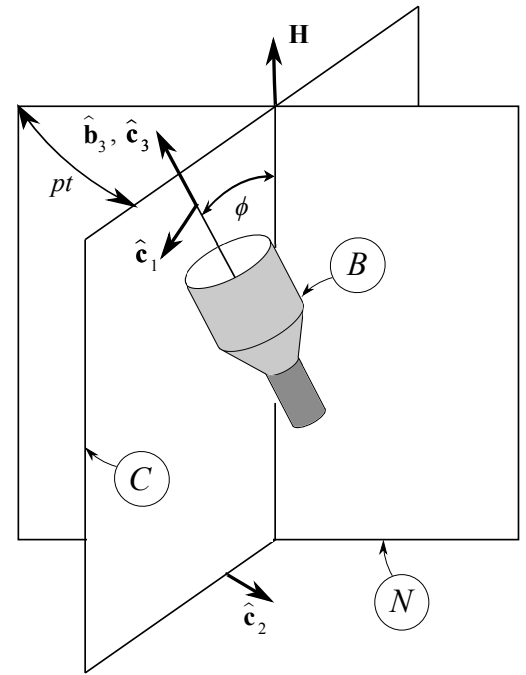

Fig. 3. Axisymmetric spacecraft, $B$, duplicating torque-free rotational motion of axisymmetric asteroid.

(see Fig. 3) can be introduced such that $\mathbf{H}$ and the asteroid's axis of symmetry are fixed in $C$. The angular velocity of $A$ in $N$ can be described simply in terms of spin about the axis of symmetry, together with precession about the angular momentum vector (see Ref. [8], Sec. 3.1). With unit vector $\hat{\mathbf{c}}_{3}$ chosen such that it is fixed in $C$ and parallel to the asteroid's axis of symmetry, the angular velocity ${ }^{C} \boldsymbol{\omega}^{A}$ of $A$ in $C$ can be written as

$$
{ }^{C} \boldsymbol{\omega}^{A}=s \hat{\mathbf{c}}_{3}
$$

where spin speed $s$ is a constant. Moreover, the angular velocity ${ }^{N} \omega^{C}$ of $C$ in $N$ is given by

$$
{ }^{N} \boldsymbol{\omega}^{C}=p \hat{\mathbf{h}}
$$

where precession speed $p$ is a non-negative constant, and $\hat{\mathbf{h}}$ is a unit vector having the same direction as $\mathbf{H}$. Hence, the angular velocity of $A$ in $N$ can be expressed simply as

$$
{ }^{N} \boldsymbol{\omega}^{A}={ }^{N} \boldsymbol{\omega}^{C}+{ }^{C} \boldsymbol{\omega}^{A}=p \hat{\mathbf{h}}+s \hat{\mathbf{c}}_{3}
$$

and the angular acceleration ${ }^{N} \boldsymbol{\alpha}^{A}$ of $A$ in $N$ is given by

$$
\begin{aligned}
{ }^{N} \boldsymbol{\alpha}^{A} & =\frac{{ }^{N}}{\mathrm{~d} t}\left(p \hat{\mathbf{h}}+s \hat{\mathbf{c}}_{3}\right)=\mathbf{0}+s^{N} \boldsymbol{\omega}^{C} \times \hat{\mathbf{c}}_{3} \\
& =s p \hat{\mathbf{h}} \times \hat{\mathbf{c}}_{3}=s p \sin \phi \hat{\mathbf{c}}_{2}
\end{aligned}
$$

where $\hat{\mathbf{c}}_{2}$ is a unit vector fixed in $C$ and normal to the plane containing $\mathbf{H}$ and $\hat{\mathbf{c}}_{3}$ (see Fig. 3). If the axes of symmetry of $A$ and $B$ are to be parallel, then $\hat{\mathbf{c}}_{3}=\hat{\mathbf{b}}_{3}$, and the central inertia dyadic of $B$ can be expressed as

$\underline{\mathbf{I}}=I_{B}\left(\hat{\mathbf{b}}_{1} \hat{\mathbf{b}}_{1}+\hat{\mathbf{b}}_{2} \hat{\mathbf{b}}_{2}\right)+J_{B} \hat{\mathbf{b}}_{3} \hat{\mathbf{b}}_{3}=I_{B}\left(\hat{\mathbf{c}}_{1} \hat{\mathbf{c}}_{1}+\hat{\mathbf{c}}_{2} \hat{\mathbf{c}}_{2}\right)+J_{B} \hat{\mathbf{c}}_{3} \hat{\mathbf{c}}_{3}$

where $J_{B}$ and $I_{B}$ are central principal moments of inertia of $B$ for, respectively, a line parallel to $\hat{\mathbf{b}}_{3}$, and any line perpendicular to $\hat{\mathbf{b}}_{3}$. If $B$ is to duplicate the asteroid's angular velocity and angular acceleration, then ${ }^{N} \boldsymbol{\omega}^{B}={ }^{N} \boldsymbol{\omega}^{A}$ and ${ }^{N} \boldsymbol{\alpha}^{B}={ }^{N} \boldsymbol{\alpha}{ }^{A}$, and the required control torque is given by

$$
\begin{aligned}
\mathbf{T} & =\underline{\mathbf{I}} \cdot{ }^{N} \boldsymbol{\alpha}^{A}+{ }^{N} \boldsymbol{\omega}^{A} \times \underline{\mathbf{I}} \cdot{ }^{N} \boldsymbol{\omega}^{A} \\
& =p \sin \phi\left[\left(J_{B}-I_{B}\right) p \cos \phi+J_{B} s\right] \hat{\mathbf{c}}_{2}
\end{aligned}
$$

The magnitude of $\mathbf{T}$ is constant. The relationship

$$
\cos \phi=\frac{J s}{(I-J) p}
$$

where $I$ and $J$ are central principal moments of inertia of the asteroid, can be used to show that $\mathbf{T}$ vanishes if the ratio of moments of inertia of the spacecraft happens to be the same as that of the asteroid; that is, if $I_{B} / J_{B}=I / J$. Otherwise, $\mathbf{T}$ is the control torque that must be applied to maintain a relative angular velocity of zero until capture takes place.

\section{DESPIN}

Once the asteroid payload has been securely captured by the spacecraft, the composite body (spacecraft and payload) undergoes de-spin to eliminate angular velocity relative to the inertial frame. For convenience $B$ will denote the newly formed rigid body. An asymptotically stable controller is designed for applying torque to drive ${ }^{N} \boldsymbol{\omega}^{B}$ to $\mathbf{0}$. No requirements are placed on the orientation of $B$ in $N$ at the time ${ }^{N} \boldsymbol{\omega}^{B}$ reaches 0 . It is assumed that the required orientation can subsequently be obtained by briefly firing RCS thrusters at the beginning and end of a slow reorientation that consumes a negligible amount of propellant.

Let $I_{i}(i=1,2,3)$ denote the principle moments of inertia of $B$, and let $\omega_{i}(i=1,2,3)$ be defined as in Eqs. (7). Assume that $n$ RCS thrusters are mounted to the spacecraft, and each is capable of producing a moment about the center of mass of $B$. Let $F=\left[F_{1}, F_{2}, \ldots, F_{n}\right]$ be a $3 \times n$ matrix, the columns of which are the maximum moment produced by each thruster. Assume the thrusters can be throttled between 0 and 1 , and let $\eta=\left[\eta_{1}, \eta_{2}, \ldots, \eta_{n}\right]^{T}$ denote the $n \times 1$ matrix of the thruster throttles. In addition, it is assumed that the RCS thrusters are configured in such a way that when all thrusters are firing at full throttle, the net moment equals to zero; i.e.,

$$
F 1=0
$$

where $\mathbf{1}=[1,1, \ldots, 1]^{T}$ is the $n \times 1$ array with all elements equal to 1 . This is a typical RCS thruster configuration in practice. For example, RCS thrusters are often arranged in pairs, each of which applies a force couple.

Euler's dynamical equations of rotational motion for rigid body $B$ can be written as

$$
I \dot{\omega}=-\omega^{\times} I \omega+F \eta
$$

where $I$ is a diagonal inertia matrix of $B, \omega=\left[\omega_{1}, \omega_{2}, \omega_{3}\right]^{T}$, and $\omega^{\times}$is a skew symmetric matrix used to implement a cross product of two vectors.

Let a Lyapunov function be defined as the rotational kinetic energy $K$ of $B$ in $N$

$$
K=\frac{1}{2} \omega^{T} I \omega
$$

Hence, the time rate of change of $K$ is given by

$$
\dot{K}=\omega^{T} I \dot{\omega}=\omega^{T}(-\omega \times I \omega+F \eta)=\omega^{T} F \eta
$$

We seek a strategy for applying control torque that will completely dissipate rotational kinetic energy; that is, we wish to make $\dot{K} \leq 0$, and $\dot{K}=0$ if and only if $\omega=0$. 
A fast strategy would be a bang-bang profile for throttle. That is,

$$
\eta_{i}=\left\{\begin{array}{ll}
1 & \text { if } \omega^{T} F_{i}<0 \\
0 & \text { if } \omega^{T} F_{i} \geq 0
\end{array} \quad(i=1,2, \ldots, n)\right.
$$

This strategy, though effective in rapidly dissipating $K$, incurs tremendous chattering especially when $\omega$ approaches 0 . Thus, a smooth controller is desired. To this end, we seek a controller that not only dissipates $K$, but also applies torque sparingly so as to avoid excessive expenditure of propellant. These considerations lead us to an objective function $J$ defined as

$$
J \triangleq \omega^{T} F \eta+\frac{1}{2} \eta^{T} R \eta
$$

which will be minimized at each instant of time. Here $R>0$ is an $n \times n$ positive definite matrix. The optimal matrix $\eta$ can be found by setting the partial derivative of $J$ with respect to $\eta$ equal to zero; i.e.,

$$
\frac{\partial J}{\partial \eta}=\omega^{T} F+\eta^{T} R=0
$$

which leads to

$$
\eta=-R^{-1} F^{T} \omega
$$

Substituting $\eta$ into Eq. (33) yields

$$
\dot{K}=-\omega^{T} F R^{-1} F^{T} \omega \leq 0
$$

and $\dot{K}=0$ if and only if $\omega=0$. Thus, according to Lyapunov theory (Ref. [9]) the controller in Eq. (37) asymptotically stabilizes the angular velocity, and minimizes the control energy simultaneously.

Recall that the thruster throttles should be bounded between 0 and 1; however, this is not guaranteed in Eq. (37). We now proceed to modify Eq. (37) to satisfy the throttle constraint and maintain asymptotic stability. To this end, let $\eta_{\min }$ be the minimum of all throttles calculated by Eq. (37). If $\eta_{\min }<0$, then we modify $\eta$ as

$$
\eta=-R^{-1} F^{T} \omega-\eta_{\min } \mathbf{1}
$$

Now, let $\eta_{\max }$ be the maximum of all throttles as given in Eq. (39), and if $\eta_{\max }>1$, then we modify $\eta$ by scaling it by $1 / \eta_{\max }$; i.e.,

$$
\eta=\frac{-R^{-1} F^{T} \omega-\eta_{\min } \mathbf{1}}{\eta_{\max }}
$$

It can be deduced that the throttles given in Eq. (40) are all between 0 and 1 by construction. Substitute this controller into Eq. (33), and we get

$$
\begin{aligned}
\dot{K} & =\omega^{T} F \frac{\left(-R^{-1} F^{T} \omega-\eta_{\min } \mathbf{1}\right)}{\eta_{\max }} \\
& =-\frac{1}{\eta_{\max }} \omega^{T} F R^{-1} F^{T} \omega \\
& \leq 0
\end{aligned}
$$

\begin{tabular}{|c|c|c|}
\hline Pod & Position (m) & Thrust Directions \\
\hline$P_{1}$ & $\mathbf{r}^{B^{*} P_{1}}=2 \hat{\mathbf{b}}_{1}-2.65 \hat{\mathbf{b}}_{3}$ & $\pm \hat{\mathbf{b}}_{2}, \pm \hat{\mathbf{b}}_{3}$ \\
\hline$P_{2}$ & $\mathbf{r}^{B^{*} P_{2}}=-2 \hat{\mathbf{b}}_{1}-2.65 \hat{\mathbf{b}}_{3}$ & $\pm \hat{\mathbf{b}}_{2}, \pm \hat{\mathbf{b}}_{3}$ \\
\hline$P_{3}$ & $\mathbf{r}^{B^{*} P_{3}}=2 \hat{\mathbf{b}}_{2}-2.65 \hat{\mathbf{b}}_{3}$ & $\pm \hat{\mathbf{b}}_{1}, \pm \hat{\mathbf{b}}_{3}$ \\
\hline$P_{4}$ & $\mathbf{r}^{B^{*} P_{1}}=-2 \hat{\mathbf{b}}_{2}-2.65 \hat{\mathbf{b}}_{3}$ & $\pm \hat{\mathbf{b}}_{1}, \pm \hat{\mathbf{b}}_{3}$ \\
\hline
\end{tabular}

and $\dot{K}=0$ if and only if $\omega=0$. Here, the assumption in Eq. (30) is used. Thus, the controller in Eq. (40) asymptotically stabilizes the angular velocity.

Remark 1: As $\eta_{\min }$ is subtracted from each throttle in Eq. (39), some thrusters may be fired unnecessarily, resulting
TABLE I

RCS THRUSTER CONFIGURATION

in needless propellant consumption. The thruster firings commanded by Eq. (39) that produce no resultant torques need to be eliminated. For example, if thrusters $i$ and $j$ with the same maximum thrust produce moments in opposite directions, and they are commanded to throttle $\eta_{i} \geq \eta_{j}>0$, then both thrusters are fired unnecessarily. These throttles can be clipped by setting the new $\eta_{i}$ to $\eta_{i}-\eta_{j}$, and the new $\eta_{j}$ to 0 . Doing so does not change the commanded torque, and thus does not affect the stability of the closed-loop system.

Remark 2: The special case where the thrusters are arranged in collocated pairs, each of which provides thrust in opposite directions, can be equivalently described as having $n / 2$ thrusters with throttles between -1 and 1 . Let $\eta_{\max }^{\prime}$ denote the maximum absolute value of the throttles given in Eq. (37). If $\eta_{\max }^{\prime}>1$, then the controller in Eq. (37) can be modified to satisfy the throttle bounds as

$$
\eta=-\frac{R^{-1} F^{T} \omega}{\eta_{\max }^{\prime}}
$$

It is straightforward to see that the asymptotic stability of the controller is maintained.

\section{Numerical EXAMPles}

In this section, numerical examples will be presented to show the effectiveness of the controllers. The spacecraft design is adopted from Ref. [1]. The mass of the spacecraft is $18,000 \mathrm{~kg}$. The cylindrical bus is axisymmetric, with a diameter of $2.7 \mathrm{~m}$ and a height of $5.9 \mathrm{~m}$. Four pods, each containing four RCS thrusters, are mounted to the bus on struts as shown in Fig. 5 of Ref. [1] (p. 23). Each pod has a radial distance of $2 \mathrm{~m}$ from the cylinder's axis of symmetry, and is $0.3 \mathrm{~m}$ from the cylinder's end. Each thruster can apply $200 \mathrm{~N}$ of force, and has a specific impulse Isp $=287 \mathrm{~s}$. The position vector $\mathbf{r}^{B^{*} P_{i}}$ from the mass center of the bus, $B^{*}$, to each of the four pods $P_{i}(i=1,2,3,4)$, and directions of thrust, are reported in Table 1.

\section{A. Example 1: Attitude Synchronization}

Use of the feedback controller to align $\hat{\mathbf{b}}_{3}$ with $\hat{\mathbf{a}}$ is demonstrated in an example involving a tumbling unsymmetric asteroid, taken to be an ellipsoid with semidiameters $\alpha=5 \mathrm{~m}, \beta=3 \mathrm{~m}$, and $\gamma=4 \mathrm{~m}$, and a uniform density of $2000 \mathrm{~kg} / \mathrm{m}^{3}$. The angular velocity of $A$ in $N$ is characterized at $t=0$ by the values ${ }^{N} \boldsymbol{\omega}^{A} \cdot \hat{\mathbf{a}}_{1}=0.6$ $\mathrm{deg} / \mathrm{s},{ }^{N} \boldsymbol{\omega}^{A} \cdot \hat{\mathbf{a}}_{2}=0.6 \mathrm{deg} / \mathrm{s}$, and ${ }^{N} \boldsymbol{\omega}^{A} \cdot \hat{\mathbf{a}}_{3}=6.0 \mathrm{deg} / \mathrm{s}$ (60 revolutions per hour). The line of descent, $\hat{\mathbf{a}}$, is chosen to have the same direction as $\hat{\mathbf{a}}_{3}$.

Although four controllers are presented in Section II-B, only the linear controller in Eq. (18) is demonstrated here. 
It is assumed here that the procedure of reorienting the spacecraft and spinning up about the axis of symmetry is executed before $t=0$. That is, at $t=0, \hat{\mathbf{b}}_{3}$ is aligned with $\hat{\mathbf{a}}_{3}$, and the spacecraft spins about $\hat{\mathbf{b}}_{3}$ at $6.0 \mathrm{deg} / \mathrm{s}$. In addition, the angle between $\hat{\mathbf{b}}_{1}$ and $\hat{\mathbf{a}}_{1}$ is arbitrarily chosen to be $60 \mathrm{deg}$. The controller consisting of Eqs. (18), (20), and (22) is applied at $t=0$.

Results are shown in Fig. 4. Figure 4(a) shows the measure numbers $\delta_{i}(i=1,2,3)$ of the angular velocity of the spacecraft relative to the asteroid. The controller is able to drive the errors to zero in about $200 \mathrm{~s}$. The angle between the spacecraft axis of symmetry and the line of descent, which can be expressed as $\cos ^{-1}\left(\hat{\mathbf{b}}_{3} \cdot \hat{\mathbf{a}}_{3}\right)$, is shown in Fig. 4(b). The angle is driven to zero within about $200 \mathrm{~s}$. The torque commanded by the controller is shown in Fig. 4(c). Notice that after $200 \mathrm{~s}$ torque is required continuously to keep the angular velocity errors at zero, and $\hat{\mathbf{b}}_{3}$ aligned with $\hat{\mathbf{a}}_{3}$.

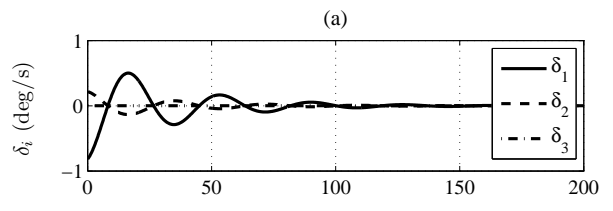

(b)

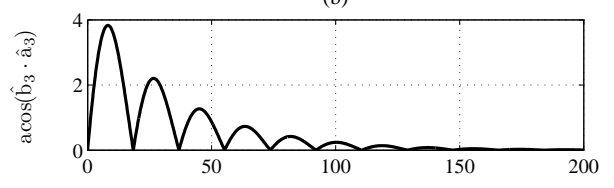

(c)

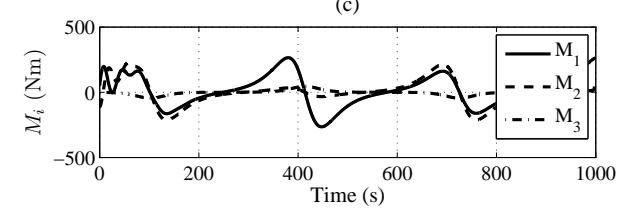

Fig. 4. (a) Relative angular velocity measure numbers $\delta_{i}={ }^{A} \boldsymbol{\omega}^{B} \cdot \hat{\mathbf{b}}_{i}$ ( $i=1,2,3$ ), (b) angle between the axis of symmetry and the line of descent, (c) control torque required for attitude synchronization.

\section{B. Example 2: Despin}

The despin controller is demonstrated with the spacecraft and asteroid introduced in Example 1. The result of capture is that $\hat{\mathbf{b}}_{3}$ has the same direction as $\hat{\mathbf{a}}_{3}$, the top of the spacecraft is in contact with the asteroid, and a 60-deg relative angle about $\hat{\mathbf{b}}_{3}$ is present, as discussed in Example 1. Unit vectors $\hat{\mathbf{b}}_{1}, \hat{\mathbf{b}}_{2}$, and $\hat{\mathbf{b}}_{3}$ fixed in the composite body $B$ are chosen to be parallel to the diameters of the ellipsoidal asteroid. The angular velocity of $B$ in $N$ is characterized at $t=0$ by the values ${ }^{N} \boldsymbol{\omega}^{B} \cdot \hat{\mathbf{b}}_{i}(i=1,2,3)$ equal to $0.6 \mathrm{deg} / \mathrm{s}, 0.6 \mathrm{deg} / \mathrm{s}$, and $6.0 \mathrm{deg} / \mathrm{s}$, respectively.

Because the mass of the captured asteroid is significantly greater than that of the spacecraft, the center of mass of $B$ is close to that of the asteroid. In this case, moment in the direction of $\hat{\mathbf{b}}_{1}$ or $\hat{\mathbf{b}}_{2}$ is applied with an arm that is 4.2 times as large as the arm associated with moment in the direction of $\hat{\mathbf{b}}_{3}$. In Ref. [2], tumbling is intentionally induced in order to take advantage of larger moment arms. In the present example, the largest component of angular velocity is in the

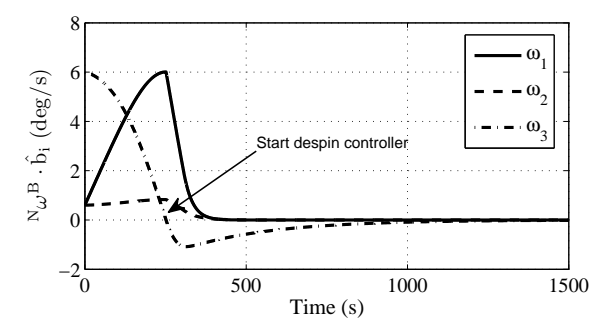

Fig. 5. Angular velocity of the composite body during despin.

direction of $\hat{\mathbf{b}}_{3}$, which is parallel to the intermediate axis of inertia, and the initial conditions correspond to a state of tumbling. By waiting for $251.7 \mathrm{~s}$ until the angular momentum is transferred to the plane spanned by $\hat{\mathbf{b}}_{1}$ and $\hat{\mathbf{b}}_{2}$, and then applying the despin controller, one can take advantage of the larger moment arms. The controller drives the angular velocity asymptotically to zero, as shown in Fig. 5, and $34 \mathrm{~kg}$ of propellant in consumed. The cost of not waiting for angular momentum transfer is $64 \mathrm{~kg}$ of propellant, an increase of $88 \%$.

\section{CONCLUSION}

Attitude control associated with capturing a free-flying asteroid using an axisymmetric spacecraft is considered. It has been shown that the axis of symmetry of the spacecraft can be aligned asymptotically with a line of descent fixed in an asteroid, and the relative angular velocity between the two bodies can be made to vanish. An analytical expression is obtained for the torque required to keep the spacecraft axis of symmetry aligned with that of an axisymmetric asteroid. After the asteroid is securely captured, the angular velocity of the composite body relative to an inertial frame can be driven asymptotically to zero. In some cases propellant consumption can be reduced by waiting for angular momentum transfer to occur before applying the despin controller, because then advantage can be taken of longer momentum arms about certain axes.

\section{REFERENCES}

[1] Brophy, J., et al., "Asteroid Retrieval Feasibility Study," Keck Institute for Space Studies, Pasadena, California, April 2, 2012. http://kiss.caltech.edu/study/asteroid/asteroid_final_report.pdf

[2] Roithmayr, C. M., Shen, H., Jesick, M., and Cornelius, D. M., "Catching a Rolling Stone: Dynamics and Control of a Spacecraft and an Asteroid," IAA-PDC13-04-27, 2013 Planetary Defense Conference, Flagstaff, AZ, April 15-19, 2013.

[3] Roithmayr, C. M., Shen, H., Jesick, M., and Cornelius, D. M., "Docking, Grappling, Capture, Control, and Alternative Approaches," Target NEO 2 Workshop, Washington D.C., July 9, 2013.

[4] Tsiotras, P., and Longuski, J. M., "A New Parameterization of the Attitude Kinematics," The Journal of the Astronautical Sciences, Vol. 43, No. 3, 1995, pp. 243-262.

[5] Tsiotras, P., and Longuski, J. M., "Spin-Axis Stabilization of Symmetric Spacecraft with Two Control Torques," Systems and Control Letters, Vol. 23, No. 6, 1994, pp. 395-402.

[6] Kane, T. R., and Levinson, D. A., Dynamics: Theory and Applications, McGraw-Hill, New York, 1985.

[7] Hudson, R. S., and Ostro, S. J., "Shape and Non-principal Axis Spin State of Asteroid 4179 Toutatis," Science, Vol. 270, No. 5233, 1995, pp. 84-86.

[8] Kane, T. R., Likins, P. W., and Levinson, D. A., Spacecraft Dynamics, McGraw-Hill, New York, 1983.

[9] Slotine, J.-J. E., and Li, W., Applied Nonlinear Control, Prentice-Hall, Inc., Englewood Cliffs, New Jersey, 1991. 